\title{
Ether and Relativity
}

\author{
Mehrdad Farhoudi* and Maysam Yousefian ${ }^{\dagger}$ \\ Department of Physics, Shahid Beheshti University, Evin, Tehran 19839, Iran
}

November 29, 2015

\begin{abstract}
We consider one of the fundamental debates in performing the relativity theory, namely, the ether and the relativity points of view, in a way to aid the learning of the subjects. In addition, we present our views and prospects while describing the issues that being accessible to many physicists and allowing broader views. Also, we very briefly review the two almost recent observations of the Webb redshift and the ultra high-energy cosmic rays, and the modified relativity models that have been presented to justify them, wherein we express that these justifications have not been performed via a single model with a single mechanism.
\end{abstract}

PACS number: 03.30. $+p ; 95.85 . R y ; 04.20 . C v ; 98.80 . J k$

Keywords: Ether Theory; Relativity Theory; Absolute Space; Lorentz Violation; Varying Speed of Light; Doubly Special Relativity.

In commemorating the first century of the discovery of general relativity by Albert Einstein that was recognized as a triumph of the human intellect, it would be instructive to look through one of its fundamental debates, namely, between the ether and the relativity points of view. Certainly, very vast amount of work have been performed on these subjects and the references given in this compact survey are not obviously a complete bibliography on these topics, and although we provide adequate references, it is a self-contained work. However, while we are trying to spell out some basic issues behind the subject, the work almost provides a brief review in a different perspective about the long history and the situations of the ether and relativity up to the present days. Nevertheless, it has not been only aimed to give just a motivation to research on the issue, and we propose, somehow during the work, to introduce our points of view and prospects on these subjects in a way that being accessible to many physicists and allowing broader views.

It seems that it was Descartes who first introduced into science the concept of ether as a spacefilling material in the manner of a container and a transmitter between distant bodies (similar to what that, nowadays, we call it a field) in the first half of seventeenth century [1. About one generation after him, perhaps one can consider Newton as one of the ether theory pioneers who practically introduced ether into physics. Actually, Newton presented the concept of inertia in the first law (i.e., the inertia law) of his famous three laws of mechanics [2], and in this respect, he considered an inertial frame as a rigid frame in which free particles move with constant speed in straight lines. On the other hand, a free particle is a particle that moves with constant speed in a straight line in an inertial frame; and obviously, this is a vicious circle (or, a logical loop). In another word, it is ambiguous that what thing distinguishes or singles out the class of inertial frames as criteria or standards of non-acceleration from all other frames. Newton, who was also aware of this difficulty, in order to specify the inertial frames from theoretical point of view, employed the idea of absolute

\footnotetext{
*E-mail: m-farhoudi@sbu.ac.ir

${ }^{\dagger}$ E-mail: M_Yousefian@sbu.ac.ir
} 
space with the aid of the notion of ether. He considered absolute space as a rest inertial frame (or the Newtonian ether) that actually is a very thin motionless media with nearly zero density, perfect luminosity and strong elasticity character, which is also a conveyer for force transmission. To Newton's contemporaries, like Hooke and Huyghens, the ether's main function was just to carry light waves and thus, it could also be acted on 11. However, the Newton idea on the ether based on it as an acting substance which does not accept reaction, but Leibniz (or, Leibnitz) insisted that the space is an order of coexistence 1 [ [5, 6. He argued against the Newton idea of substantival ontology of space, and believed that this idea leads to contract with the principle of sufficient reason [7]. Also, Berkley presented some arguments against the Newton absolute space on his work named De Motu (On Motion) [8]. However, when the luminiferous ether evolved into a cornerstone of the Maxwell theory [9], it became a plausible marker for the Newton absolute space.

In Newtonian physics, space is a pre-existing stage on which material particles are the characters acting out the drama of physical events. This point of view is on the contrary to the Aristotelian 2 view that space is a plenum (i.e., occupied by matter) and inseparably associated with the material substance [11, 12]. In fact, the Newton view is a return to the Democritus view that space is a void with the properties which are independent of the material bodies that move in it [10]. While in relativistic gravitational physics, again, space cannot be considered apart from the matter that is in it, and, as the mathematician E.T. Whittaker [10] points out, in this case, the characters create the stage as they walk about on it, i.e., gravitation has become part of the stage instead of being a player. In another word, the properties of space in gravitational theories are inseparable from the matter that is in it. Indeed, it has been pointed out [13] that a basic problem of Newtonian mechanics is that the extrinsic state of a point particle, i.e. its appearance in space and time (that usually characterized by its position and velocity), is a priori independent of its intrinsic state (that usually characterized by its mass). However in quantum physics, each coordinate (or in another word, position that is the notion of geometry) does not commute with its corresponding momentum (or in another word, dynamics that can be considered as the notion of, moving, mass); or in other words, for each object, these two characters are not simultaneously compatible from an observer view. That is, analogous to the complementarity principle of the particle-wave duality, the issue may be interpreted as in confrontation with everything, it either represents the aspect of geometry or the aspect of matter in one instant depending on the experimental arrangements and/or the initial conditions.

Nevertheless, and principally, the innovation of absolute space is while the Galilean transformations do not distinguish among the inertial frames as well, and thus Newton, in confronting the quarry that how absolute space can be specified, presented the famous idea of the Newton bucket from the practical point of view [14, 15]. However, the Newton bucket provides the distinction of a non-inertial frame, and does not distinguish the inertial frames from each other. That is, any curve or change in the horizontal level of the bucket water does represent the acceleration of the bucket with respect to a frame which is itself either an inertial or a non-inertial one. However, Newton accounted it with respect to an inertial frame (as a criterion to distinguish acceleration), and actually with respect to a specified inertial one, i.e., absolute space. Although in this regard, Mach also interpreted the changes with respect to the average motions of all particles in the universe (or, the distant fixed stars) [16]. Indeed, and in a more accurate expression, the Newton discussion was that any curve of the horizontal level of the water cannot be because of its relative rotation with respect to the bucket, however, Mach did consider it as the relative motion between them [17]. Incidentally, and up to the available experiments, one may also not being able to locally detect an accelerated frame in the large scales, e.g., the rotation of the earth around the sun and or the rotation of the solar system around the center of our local galaxy, merely by the idea of the Newton bucket.

Before we continue our discussions, it would be also instructive to review the following well-

\footnotetext{
${ }^{1}$ This point of view is a return to the Aristotle idea. Incidentally, this idea is related to the relational physics [3, 4], which means a physical system is in a way that positions and other properties of things have meanings just with respect to the other things. This point of view is a prelude to the Mach ideas, particularly the weak version of it.

${ }^{2}$ The Aristotle point of view on space was asserted in his definition of place as the limit between the surrounding and the surrounded body [10, and also as the innermost motionless boundary of that which surrounds it [11, 12.
} 
known proposed experiment on the issue. Consider the rotation of the plane of a swinging Foucault pendulum at the earth's north pole. Within the limits of experimental accuracy $3^{3}$ the remarkable fact is that the times taken for the earth to rotate a complete round with respect to absolute space, and relative to the fixed stars are the same. In Newtonian view, there is nothing a priori to predict this result, and it is just a coincidence. In other words, the result indicates that the fixed stars are not rotating (or, do not have acceleration) relative to absolute space, and can be employed as a criterion to specify the class of inertial frames. However in Machian view, one precisely expects that the two time durations of the measurements must be the same regardless of the accuracy of the instruments, for, in his view, the detected criteria of acceleration are exactly the fixed stars.

In the historical process, Maxwell offered an another way to specify the Newton ether. In the ether theory presented by him [9], absolute space is accounted as a media for the light propagation, and is specified via the Maxwell equations (or from his point of view, the ether equations). Regarding this theory, due to the Fresnel dragging effect [23, 24], the speed of light in the other inertial frames is different from the ether one, as the Maxwell equations are not invariant under the Galilean transformations. To investigate the Maxwell ether, the Michelson-Morley experiment [25]-[27] was performed in the year of 1887, and the Maxwell ether was not confirmed. However, to explain the null result of the Michelson-Morley experiment, Fitzgerald proposed a hypothesis in the year of 1889 [28]. According to his hypothesis, when a body moves with a constant velocity with respect to the ether, it will be (really) contracted in the direction of motion.

Lorentz, like Maxwell, believed that the light propagation, similar to the sound, requires a media which is the ether as the characteristic of absolute rest. Thus, he also proposed [29, 30] a hypothesis (although independent, but actually an elaboration of the Fitzgerald hypothesis) to explain the Michelson-Morley experiment. According to the Lorentz ether theory [31, a body with a constant velocity with respect to the ether is also contracted 4 in the direction of motion (the Lorentz-Fitzgerald contraction) and its clocks are slowed down [32] (i.e., the clock retardation and/or (real) time dilation) when moving through the ether. By his theory, the results of the Michelson-Morley and even the Kennedy-Thorndike [33] experiments, which is a broader and more general one than the previous experiment, are also explained. Incidentally, based on the Lorentz ether theory, and similar to the Newtonian mechanics, the inertial reference frames are related to each other by the Galilean transformations, however according to the Lorentz-Fitzgerald contraction hypothesis, the form of the Maxwell equations still remain invariant. In this case, as the earth is surrounded by the ether, there are two choices. Either the ether must be dragged by the earth and remains at rest with respect to it, which the aberration observation [34, 35] has rejected this choice. Or, the ether must not be dragged by the earth and contains a velocity with respect to it, in a way that the Fresnel dragging should be observed for light. This subject was investigated via the Fizeau experiment [36]-38], and the Lorentz ether theory has also been able to explain the result of this experiment by the aid of local time dilation hypothesis. Regarding these facts, there have been some debates, comparisons and investigations about the originality and the equivalence of the Lorentz ether theory with the special theory of relativity (STR); see, e.g., Refs. [1, 39]-[43] and references therein. However, the two theories are logically independent, because obviously, the choice of different postulates principally leads to theories which differ in their simplicity and appeal, although they may observationally be equivalent.

However, Einstein, and Mach before him, were also among the opponents of the Newton absolute space, and in this regard, they raised two main objections. First, how absolute space, as an inertial frame, can be theoretically distinguished and located from all other inertial frames in a unique way. Second, how absolute space can act on every particle and distinguishes free particles from other ones, but cannot be acted upon. In general, from the Einstein point of view, the existence of a matter

\footnotetext{
${ }^{3}$ Also, the Lense-Thirring precession effect (see, e.g., Refs. [18]-20]), or actually the frame-dragging effect [21, 22], must be neglected.

${ }^{4}$ Such a contraction was accounted in terms of the Lorentz electron theory 31; however, it is believed that some other results predicted from his theory could not be found experimentally [24] and the theory has some philosophical deficit such that its basic assumptions are unverifiable [14.
} 
which is completely transparent, its nature is unspecified and obscure, and there is no way to prove its existence.5 was not required. Eventually, in 1905, Einstein by proposing the STR [48], attracted most of the attentions towards this theory. Actually, Einstein presented his theory by generalizing the Newton absolute space to the Minkowski spacetime [49] and extending Newtonian relativity with the Galilean transformations to special relativity with the Lorentz transformations, and could explain the whole mentioned experiments very well. However in the STR, the inertial frames, as the preferred ones, are still the references and the criteria for the absoluteness of the concept of acceleration 6 and yet, the difficulty remains in theoretically distinguishing these class of frames from all other frames. Indeed, the Galilean relativity principle, in general, contains the four-dimensional special relativity formulation [52. Nevertheless, in the STR, as the equivalence of the inertial frames are valid for all physical laws (including the Maxwell equations), the Maxwell ether hypothesis is rejected. However, it cannot verify absolute space (although, still has the mentioned objections to it), but it cannot also deny its existence even though absolute space cannot be distinguished by intrinsic properties from all other inertial frames. On this point of view, Einstein was not satisfied with the theory too.

It may be surprising, but, perhaps due to some points (like the mentioned ones), even Mach, whose critiques to the Newtonian mechanics paved the way to the relativity theory smooth (at least philosophically), was suspicious on the Einstein theory? However, due to many experimental verifications of the STR obtained from the wide diversity of different phenomena, the skeptics had to give up; indeed, special relativity probably is the most based and reliable tested theory in the contemporary physics. Nevertheless and despite the enormous experimental robustness on the STR, in the last two decades, due to the theoretical posited questions, scientists are eagerly looking for experimental findings that somehow violate the STR [53]. In this regard, the researches are particularly focused on experiments that indicate violation of the Lorentz symmetry 8 . However, and as a rough estimation, the quantum gravity induced Lorentz violation can only be achieved as a theoretical purpose, for the natural scale that one would expect (in this respect and as a strong violation) is the Planck energy of about $10^{19} \mathrm{GeV}$, while the highest known energy particles is the ultra high-energy cosmic rays (UHECR) [55-57] of about $10^{11} \mathrm{GeV}$ and the present accelerator energies are about $10^{3} \mathrm{GeV}$ that preclude any direct observation of the Planck scale Lorentz violation. Nevertheless and in particular, some physicists have looked for evidence on invalidity of the principle of relativity [58], or for deriving special relativity from Galilean mechanics alone [59] and or for testing the STR by investigating what sort of new bounds can be achieved at high energies while the Lorentz symmetry is not satisfied [60].

On the other hand, on the way to reject absolute space and absolute concept of acceleration, Einstein himself also attempted to propose the general theory of relativity (GTR) in 1915 [15, 61, 62. inspired from the Mach ideas 1, 17, 63) and with the aid of the principle of equivalence of gravitation and inertia [14, 64, 65] as a clue principle9. Of course, in the GTR, no way or solution has also been provided for determining the inertial frames, and in fact in this theory, no preference or reference, as a criterion of non-acceleration, is taken to these frames as well. That is, it is appeared that in the GTR, the question of how to determine the inertial frames has been deleted. Nonetheless, in the GTR, contrary to the strong version of the Mach principle (i.e., space is not expressed as an independent essence/substance, but merely as an abstraction from the totality of distance-relations

\footnotetext{
${ }^{5}$ However, nowadays, the discovery of the acceleration of the universe [4]-47] can be discussed as a possible way of investigating the contrary to such a claim.

${ }^{6}$ Thus, the Newton first law is consistent with special relativity. However, for distinguishing the inertial frames from the rigid frames, instead of the existing context of the Newton first law, one can employ, e.g., the law of light propagation. In fact, the usual definition of rigid bodies cannot be applied in special relativity, although to be consistent with it, some new definitions have been stated. For instance, the characteristic of rigidity is assigned to a body as relative-rigidity that any length element of the body on the move remains invariant with respect to the comoving observer [50] or a body on the move somehow deforms continuously that each of its infinitesimal elements has just the Lorentz contraction with respect to the instantaneous rest observer [51.

${ }^{7}$ His critical view on the STR has been explicitly expressed in the foreward to the ninth edition of his book [16].

${ }^{8}$ Among the reviews on the Lorentz violation, Ref. [54] includes the theoretical approaches as well as the phenomenological analysis.

${ }^{9}$ Meanwhile, for grasping more about the contents and points that led to the advent of the GTR; see Refs. [66]-70].
} 
between matter), the spacetime is attained as an independent essence/substance which both acts on the matter and is reacted upon (indeed, it has the weak version of the Mach principle). Meanwhile, the confusion surrounding the principle of equivalence led a physicist like Synge to suggest, in the preface of his book about the GTR 71, that this principle has to be set aside and the facts of absolute spacetime be faced. Although, to clear some of the ambiguities about the principle of equivalence, it is emphasized that its statement must be stated locally, wherein by locally it is meant a region over which the variation of the gravitational field cannot be detected [14]. Nonetheless, even with this type of statement, it seems that still the ambiguities have not been completely eliminated.

However, about the two main raised objections on absolute space, in the GTR for elimination of the first objection, there is no need or preference requirement to propose an absolute space. Resolution of the second objection is also expressed by accepting an independent essence (or, substance) for geometry (i.e., space), and actually, by appealing to the weak version of the Mach principle. On the other hand, while forming the GTR, it was specified [68 via the hole argument (or, problem) 11 that the point events of the spacetime manifold had been incorrectly thought of as individuated independently of the field itself. That is, it is impossible to drag the metric field away from a physical point in empty spacetime and leave that physical point behind. As Einstein himself wrote [76, 77] that nothing is physically real but the totality of spacetime point coincidences, and placed [78] great stress on the inseparability of the metric and the manifold. Hence, the spacetime continuum (i.e., physical events) is the same as space points (or, manifold) that are not separated from the metric (i.e., geometry of space). 12 In fact, a key lesson raised from the Einstein gravitational theory is that the gravitational field has been inseparably twisted/intricated with the geometry of spacetime, and thus, geometry itself is an impellent essence (or, dynamic).

In this regard, it is worth to mention that most of the leading relativists in the early twentieth century, for examples, Eddington [79] and even Einstein himself [80, claimed that, in principal, the GTR is merely an ether theory 13 On this issue, Trautman has asserted [81] that he has presented the mathematical demonstration of such a claim by obtaining a form of the GTR without spatial curvature. And recently, by employing a combination of Lorentz's and Kelvin's conception of the ether 14 and actually by using the Lorentz-Kelvin ether theory [40, 82, 83], the Einstein field equations has been obtained [84.15. Meanwhile, it has also been claimed in Ref. 85] that there is an underlying relationship between the GTR and Newton's absolute time and space (via the existence of a preferred set of coordinates in general relativity 16 that is equivalent to Newton's absolute time and space). And even it has been asserted [86] that, in terms of the Newton laws within 4-dimensional curved geometries, the GTR can be exactly described.

Also in relevance to the Mach ideas, some physicists just believe that, in his ideas, the inertial frames have been replaced by the average motions of all particles in the universe and the influence of distribution of matter in the immediate vicinity of any particle, as well as the other distant bodies, determines the inertial frames. Nonetheless, in this kind of belief, this new reference adapts its nature from the whole matter of the universe. On the other hand, in a few recent decades, some novel ideas and theories have been proposed as "geometrical description of physical forces", "geometrical base

\footnotetext{
${ }^{10}$ Principally in gravitational physics, energy itself acts as a source of gravity and is not capable of simply being thrown away, and also one cannot easily rescale the zero point of it [72, 73].

${ }^{11}$ It had been thought that generally invariant field equations cannot uniquely determine the gravitational field generated by certain distributions of source masses, in contradiction with the requirement of physical causality [14, 15. 74, 75].

${ }^{12}$ This is known as the point-coincidence discussion.

${ }^{13}$ In this respect; see also Ref. [15] and references therein.

${ }^{14}$ That is, the ether as a substance of some kind, and not a type of vacuum without any properties intrinsic to itself (e.g., the ether would have the property of ponderability, which is to say, it has the power to gravitate or to generate curvature).

${ }^{15}$ By adopting that the ether gravitates only in the presence of matter.

${ }^{16}$ Note that, in general, any relativistic gravitational equation, including the Einstein equations of the GTR, is needed to be non-linear, and hence, its number of independent solutions are not finite and the superposition principle is not also valid for it.
} 
of material content of the universe", "geometrical curvature induces matter" and "induced-matter theory" which usually connect extra dimensions (i.e., geometry) to the properties of the matter [87][96. Even some gravitational theories have been considered in which the Lagrangian of the geometry, that is usually supposed to be the characteristic of the geometry alone, just from the beginning, and indeed a priori, is presented as a function of the geometry and the matter [97]-[105]. Now, inspiring from these types of ideas and theories, and knowing that, in the Mach ideas, the inertia of a body is not just the intrinsic property of that body, but is caused by the cosmic masses via some interactions (where the influence of the distant bodies preponderates) 17 one can perhaps relate this cause to be due to the cosmic background (e.g., the whole geometry of the universe).

Nevertheless, to make the GTR more consistent with even the strong version of the Mach principle, Einstein inserted [106] the well-known term of the cosmological constant 19 into his equations However, when de Sitter achieved [107] his solution for the vacuum GTR plus the cosmological constant term 21 Einstein vehemently retook the inclusion of such a term while describing it as the biggest mistake he ever made [109]. Even in this regard, realizing that the metric field is not a phenomenon resulted of matter but has its own independent existence, Einstein, near the end of his life, gradually decreased his enthusiasm for the Mach principles. Indeed in 1954, he wrote to Pirani that one should no longer speak of the Mach principles at all [67, 110]. Perhaps the main point of the issue roots in considering spacetime as a new inertial standard which directly influences by the active gravitational mass through the Einstein equations, although, in the absence of mass and other disturbances, still spacetime would straighten itself out into the class of extended inertial frames, contrary to the idea that all inertia is caused by the cosmic masses.

Nonetheless, by considering the necessity of conformal symmetry breaking, the inclusion of the cosmological constant term is still proposed to remedy the inconsistency of the Einstein gravitational theory with the strong version of the Mach principle [111]. On the other hand, besides confronting the cosmic gravitational collapse (due to gravity among them), there needs to have a kind of repulsing force for explaining the recent discovered acceleration of the universe [44]-[47], which seems to originate from "property" of geometry itself or spacetime in global scales, contrary to the well-known forces up to now. In this respect, the Einstein equations including the cosmological constant term have again been considered, and this term is interpreted as if the vacuum fluid and the vacuum energy density 22 see, e.g., Refs. [118, 119]. Incidentally in this regard, the ether energy-momentum tensor introduced in Ref. [84] is not dissimilar to this term. Also in the last two decades, in the dark energy issue [120]-[125] (an energy that consists nearly $69 \%$ of the matter density of the universe [126, 127]), it seems as if the geometry (or in another word, space) in the cosmological scale has an anti-gravity type of interaction. In essence, in these ideas, both the geometry and matter (in its general meaning,

\footnotetext{
${ }^{17}$ However in the Mach ideas, there is also no description about why the interaction should be velocity-independent, but acceleration-dependent, and or indeed, why there is such a distinction between unaccelerated and accelerated motion in the nature.

${ }^{18}$ Incidentally, by this insertion, he also provided the possibility of having a static solution for the universe (that was thought to be so at that time), as an appropriate condition on the GTR.

${ }^{19}$ Even a sufficiently small value of the cosmological constant can have very important effects on the evolution of the universe; and although the implications of this term are cosmological, the origin of it is probably to be found in the quantum theory rather than cosmology.

${ }^{20}$ The Einstein equations are $G_{\mu \nu}=\left(8 \pi G / c^{4}\right) T_{\mu \nu}$, where $G_{\mu \nu}$ is the Einstein tensor as a function of the metric and its derivatives, $G$ is the Newtonian gravitational constant, $T_{\mu \nu}$ is the energy-momentum tensor and the lower case Greek indices run from zero to three. The Einstein equations plus the cosmological constant are $G_{\mu \nu}-\Lambda g_{\mu \nu}=\left(8 \pi G / c^{4}\right) T_{\mu \nu}$, where $g_{\mu \nu}$ is the metric tensor and $\Lambda$ is a constant.

${ }^{21}$ Meanwhile and almost around the same time, non-static closed solutions of the GTR (corresponding to an expanding distribution of matter) were discovered, and also it was specified that the universe is not static, but rather is expanding in the large-scale (that was officially published a few years later 108]).

${ }^{22}$ Incidentally, according to quantum theory, the vacuum has vacuum fluctuations and an energy tensor (zero-point energy) that the only form of it (being the same in all inertial frames) is a constant multiple of the metric, i.e. the same as the cosmological constant term. However, the calculations based on theories of elementary particles yield a value for the corresponding cosmological constant term to be orders of magnitude far larger than the observations allow. This discrepancy is known as the cosmological constant problem; see, e.g., Refs. [112]-[117] and references therein.
} 
including material and radiation) are different aspects of a "thing" (or in another word, existence), although, even by accepting an independent entity for each one, they would somehow relate to the other one as well (at least, through that "thing"); see, e.g., Ref. [128] and references therein.

In addition to the dark energy issue, the other cosmological observations have indicated [129]-133] that there should also be another kind of matter besides the usual barionic matters, i.e. an exotic fluid called dark matter, that consists [126, 127] nearly $26 \%$ of the matter density of the universe. These two important cosmological problems and, on the other hand, the quantizing difficulty [72, 73, 128, 134] of the Einstein gravity (in spite of the impressive successes of it) are, in general, the main reasons that have raised the need to investigate generalized or alternative gravitational theories. In this respect, and for instance, one of the alternative theories is the Brans-Dicke gravitational theory [135] that is more consistent with the Mach ideas. In connection to our discussion, also in this theory, there is a kind of matter in the form of a scalar field in the whole space in addition to the usual matter (or, the barionic matter) [136]. Actually, while the Brans-Dicke gravity is regarded as the generalized Einstein gravity, its Lagrangian can be converted to the Einstein gravitational Lagrangian plus a scalar field term via the conformal transformation [137, 138]. Meanwhile, in the other gravitational theories of type of the Brans-Dicke gravity (or in general, the scalar-tensor gravitational theories [137, 139, 140]), in particular, the chameleonic gravitational theories [141]-144], by the coupling of a scalar field with the metric (or in another word, space), the dynamic of the scalar field depends on the surrounding background density which requires that the interaction of this scalar field with the usual matter to be of gravitational type. Among different types of the modified gravitational theories, one can also mention the Einstein-ether [54, 145]-150] (and references therein) and non-minimal æthermodified [151] gravity theories. In these theories, in general, the coupling of the Einstein gravity with a dynamical timelike vector field (representing a preferred rest frame, i.e., ether) is considered.

Essentially, one of the three probable assumptions that Brans and Dicke stated in their work [135] is that physical space has intrinsic geometry and inertial properties beyond those that can be achieved from the matter contained therein, however, in their work [135], they proceeded the other assumption that leads to the Brans-Dicke gravitational theory. Nonetheless, and also according to the Dicke view [136, the introduced scalar field in this theory is a field that along with the metric are described as the gravitational field (or in another word, geometry). In this regard, in the ancient time, although Plato did not accept the view of void space and believed that space is a plenum (i.e., a general assembly), but his view was also different from the Aristotelian one. In the Plato view, space is an entity that bodies are made out of it and cannot exist without it [10].23 In other words, Plato identified space as that in which things come to be [152, 153].

Moreover, in the last two decades, another two observations, namely the Webb redshift [154, 155] and the UHECR [55]-[57, 156], have been reported while the standard Einstein relativity theory is not capable to explain these two cosmological phenomena. Hence, it was required that some modifications and generalizations being performed on the Einstein relativity. In this respect and up to now, there have been represented several models to describe the Webb redshift, including the models for varying the constants that participate in determining the atomic structure [157]. Among these types of models, one can mention the varying electric charge [158] and the varying speed of light (VSL) [159]-164] (and references therein) models, where the comparison of these two kinds of models has also been performed in Ref. [165]. On the other side, along with theories such as the non-commutative field theory [166, 167, the most reliable models, that attempt to explain the observed UHECR, are known as the doubly-special-relativity or deformed-special-relativity (DSR) [168]-[174]. However, all the available modified models on these subjects have been unsuccessful in justifying these two phenomena via a single model with a single mechanism.

To clarify the latter expression, let us very briefly review how these modified models work. Actually, it would be instructive to represent a concise description of these two recent phenomena and an overall explanations on the VSL and the DSR regarding justifications of these two observations.

During the observations of galaxies and distant stars covering the redshift range $0.5<z<3.5$,

\footnotetext{
${ }^{23}$ This point of view seems not to be irrelevant with the strong version of the Mach principle.
} 
the Australian group of Webb noticed redshifts that can be justified with a variable fine structure constant [154, 155]. Actually, in the standard cosmology, the ratio of the cosmological redshift (due to the expansion of the universe) of the absorption lines spectra of atoms on distant galaxies to the ones of the same atoms in laboratory is predicted to be the same for different amounts of energies of the absorption bands. However, Webb et al. observed that this ratio depends on the quantum numbers and the atomic and the molecular structures of the materials that radiate the corresponding rays, and hence, the structure of the absorption bands should vary due to the redshift caused by the expansion of the universe. As in the standard cosmology, the redshift usually means distant past, thus, the explanation of such a phenomenon has been based on having different atomic structure (and hence, the absorption bands) in distant past with respect to its present structure. In this regard, among the models that aim to explain the Webb redshift, the VSL models are the best option.

In relativity area and in general, the VSL models can usually be classified into two methods. In one method, new scalar fields are added to the Einstein-Hilbert Lagrangian, and another method is mainly based on changing this Lagrangian itself. In general, the appearance of any scalar field can be performed somehow to make variation in the speed of light, for, naively, it is analogous to have light rays passing through a dielectric media. It means that the appearance of any dielectric media causes variation in the speed of light, and if there is no dielectric in the matter media, the constancy of the speed of light will be in the vacuum. Thus, in these models, the speed of light practically depends on the appearance of the scalar field, by which also, the other cosmological issues, such as inflation, flatness and dark energy, are usually described.

As a simple prototype, although general, a scalar-tensor action for the VSL models, analogous to the one used in Ref. [158], can be written as

$$
S=\int d^{4} x \sqrt{-g}\left(L^{[g]}+L^{[\psi]}+L^{[m]} e^{-2 \psi}\right),
$$

where $L^{[g]} \equiv R / 16 \pi G$ is the Einstein-Hilbert Lagrangian, $L^{[\psi]} \equiv-\omega(\psi) \partial^{\alpha} \psi \partial_{\alpha} \psi / 2+V(\psi), V(\psi)$ is a self-interacting potential and $L^{[m]}$ is the matter Lagrangian. Also, $R$ is the Ricci scalar, $\omega(\psi)$ is a varying dimensionless coupling coefficient of the scalar field $\psi, g$ is the determinant of the metric and, for simplicity, we have set the speed of light, in the absence of the scalar field, to be $c(\psi=0)=1$. Variations of this action, with respect to the metric and the scalar field, yield

$$
\square \psi=\frac{1}{\omega}\left(2 e^{-2 \psi} L^{[m]}-\frac{\omega^{\prime}}{2} \partial^{\alpha} \psi \partial_{\alpha} \psi-V^{\prime}\right)
$$

and

$$
G_{\mu \nu}=8 \pi G\left(T_{\mu \nu}^{[\psi]}+T_{\mu \nu}^{[m]} e^{-2 \psi}\right)
$$

where the prime denotes the ordinary derivative with respect to the argument, $\square \equiv ; \rho^{\rho}$ and $T_{\mu \nu}^{[i]} \equiv$ $-(2 / \sqrt{-g}) \delta\left(\sqrt{-g} L^{[i]}\right) / \delta g^{\mu \nu}$. Now, by employing the spatially flat homogeneous and isotropic metric of the Friedmann-Lemaittre-Robertson-Walker (FLRW)

$$
d s^{2}=d t^{2}-a^{2}(t)\left(d r^{2}+r^{2} d \Omega^{2}\right)
$$

that includes the scale factor $a(t)$, the Friedmann-like equation for a perfect fluid achieves as

$$
\left(\frac{\dot{a}}{a}\right)^{2}=\frac{8 \pi G}{3}\left(\rho^{[m]} e^{-2 \psi}+\rho^{[\psi]}\right),
$$

where $\rho^{[m]}$ is the matter density and with the assumption of the scalar field being also homogeneous, we have $\rho^{[\psi]}=\omega \dot{\psi}^{2} / 2+V$. At last, using the cosmological considerations, the resulted equations specify the way that the scalar field and, in turn, the speed of light vary.

Meanwhile, in the VSL models, one should note that if the metric is assumed to be

$$
d s^{2}=c^{2}(t) d t^{2}-a^{2}(t)\left(d r^{2}+r^{2} d \Omega^{2}\right)
$$


it cannot by itself being used as the variation of the local speed of light (i.e., as the one that travels along the null geodesics), and hence, as the local violation of the Lorentz symmetry. Because, the speed of light, in the absence of matter, is just a criterion of the variation of time with respect to the place, which this kind of variation does not have an interesting meaning. Indeed, the variation of the speed of light and the local violation of the Lorentz symmetry, on the Riemannian manifold, can be considered only in two cases. Either the assumption is that light rays travel on a manifold and observers are on another one, wherein this case, light rays obviously do not travel along the null geodesics of the observers. As an example of this case, we can mention the "induced-matter" models [91, 92] and some of the multi-metric models [175]. Or, as an another case, there exist some fields on the Riemannian manifold that, by interaction with light, prevent light rays traveling along the null geodesics [176]-[179].

Among the VSL models, it is worths to mention the bimetric model, e.g. Refs. [176]-[179], in which the effective metric of light and matter, $\breve{g}_{\alpha \beta}$, is distinct from the spacetime metric $g_{\alpha \beta}$ as

$$
\breve{g}_{\alpha \beta} \equiv g_{\alpha \beta}+B \partial_{\alpha} \psi \partial_{\beta} \psi
$$

where $B$ is a constant coefficient with the dimension of the inverse of the energy density. The corresponding action is

$$
S=\int d^{4} x \sqrt{-g}\left(L^{[g]}+L^{[\psi]}+\frac{\sqrt{-g}}{\sqrt{-g}} \breve{L}^{[m]}\right),
$$

where all the terms are as in action (1) except that here, $\omega$ is constant and the matter Lagrangian is a function of the effective metric $\breve{g}_{\alpha \beta}$. In this model, light rays do not travel along the spacetime geodesics, and thus, there exist local variations of the speed of light with respect to the speed of graviton [176]-179]. Then, variations of the action, with respect to the metric and the scalar field, yield

$$
\square \psi=\frac{1}{\omega}\left(B \frac{\sqrt{-g}}{\sqrt{-g}} \breve{T}^{[m] \mu \nu} \breve{\nabla}_{\mu} \breve{\nabla}_{\nu} \psi-V^{\prime}\right)
$$

and

$$
G_{\mu \nu}=8 \pi G\left(T_{\mu \nu}^{[\psi]}+\frac{\sqrt{-\breve{g}}}{\sqrt{-g}} \breve{T}_{\mu \nu}^{[m]}\right) .
$$

And again, for all the same mentioned conditions, the corresponding Friedmann-like equation for a perfect fluid is

$$
\left(\frac{\dot{a}}{a}\right)^{2}=\frac{8 \pi G}{3}\left(\frac{\sqrt{-\breve{g}}}{\sqrt{-g}} \breve{\rho}^{[m]}+\rho^{[\psi]}\right),
$$

that eventually, with the aid of the cosmological considerations, specifies how the scalar field and, in turn, the speed of light vary by the time.

As mentioned, the other important recent observation, that is considered as a challenge for the STR and the Lorentz symmetry, is the UHECR [55]-[57, 156]. At first, let us briefly describe this phenomenon. When particles usually reach the specified energies, then, due to interaction with the microwaves background (that can be the cosmic infrared background [180, 181] and or the cosmic microwaves background [182]), either would be significantly absorbed by the pair-production (like, for the high-energy photons), or their energies are reduced via the photon-pion production (like, for the ultra high-energy protons and neutrons). Hence, the ultra high-energy cosmic particles have limited life-times, and thus, can travel limited distances. In this regard, in the year 1966, in two distinct papers [183, 184, the threshold energies were specified for the distances that can be traveled by the ultra high-energy cosmic particles (depending on the amount of their energies) using the calculations based on the quantum field theory and according to the Lorentz symmetry. These threshold energies, that are derived via the STR, $E_{\text {th-SR }}$, are known as the GZK threshold after Greisen-Zatsepin-Kuzmin. According to these calculations, the threshold energy for the high-energy photons are about $10^{4} \mathrm{GeV}$, and for the ultra high-energy protons are about $5 \times 10^{10} \mathrm{GeV}[55]$. 
Nevertheless, the ultra high-energy protons and photons have been observed that their energies are more than the corresponding calculated threshold energies [55]-[57]. On the other hand, as there is no source for such ultra high-energy particles inside our galaxy, hence, these particles have, in principle, been able to travel extragalactic distances. Therefore, this observation infers more life-times for these particles than the calculated ones based on the Lorentz symmetry and the standard quantum field theory [56, 57].

To explain the observation of the UHECR, as mentioned, the most reliable approach is the DSR [168-174], wherein, inspired from the notion of quantum gravity, it contains two invariant scales, e.g., the speed of light and the Planck energy. Of course, in the realm of DSR, different models have been presented. Some of these models are based on the quantum group 24 and the noncommutative geometry [170, 172, wherein, the corresponding quantum groups are related to Hopf algebra. In these type of models, either the corresponding relation of the non-commutative geometry is one of the obtained results of the quantum groups relations [170], or based on the non-commutative geometry assumption, the corresponding relations of the quantum groups are resulted [172]. Some of the other models of the DSR are presented based on the projective linear group [169, 171, 173]. As a prototype of these models, the model of Magueijo-Smolin [169] can be mentioned, in which by substituting the Fock-Lorentz [186] inertial transformations instead of the Lorentz ones, new transformations, named Magueijo-Smolin, have been defined for the energy-momentum space. Then, by the new transformations, a specified scale of energy (for instance, the Planck energy) is set as an invariant for different inertial observations. Also, some other models of the DSR are stated based on the deformation in the generators of the Lorentz group [168, 174].

However, in general, the common key point, in all of the explaining models [166]-[174] of the observation of the UHECR, is that the linear equations of the field and also the energy-momentum are somehow replaced by non-linear ones. For instance, in Ref. [168], the dispersion relation (that usually specifies the connections between the energy, momentum and mass through the Klein-Gordon equation, and also is the indicator of the linear equation of wave) is modified by a length parameter (for instance, the Planck length, $\ell_{\mathrm{p}}$ ) as a Lorentz violation parameter. Thus, in this way, via the modified dispersion relation and the conservation laws of energy and momentum, the value of the threshold energy, $E_{\mathrm{th}-\mathrm{SR}}$, increases to a new value, $E_{\mathrm{th}}$. For example, in Refs. [168, 174], the change in the dispersion relation for an ultra-relativistic (i.e., $E \gg m$ ) particle of mass $m$ and energy $E$ has been considered, via the Lorentz violation parameter and in leading order in the Planck length, as the non-linear form

$$
E^{2} \simeq p^{2}+m^{2}+\varepsilon \ell_{\mathrm{p}} p^{2} E,
$$

where $p$ is the momentum of the particle and $\varepsilon= \pm 1$ depending on the model under consideration. Note that, as we have set $c=1=\hbar$, the Planck length has the dimension of the inverse of energy, and actually, the Planck energy is the invariant (observer-independent) maximum energy scale similar to the speed of light for the speed of particles. Also, although relation (12) is not invariant under the Lorentz transformations, but it is under a sort of amended Lorentz ones depended on the considered model of the DSR. Then, as the threshold energy of a high-energy particle is the value of the energy that the particle can interact with the microwaves background, with the aid of relation (12) and the conservation laws of energy and momentum before and after the collision, the value of the threshold energy in the case of the Lorentz symmetry, $E_{\mathrm{th}-\mathrm{SR}}$, is amended up to the first-order in the Planck length to be 174

$$
E_{\mathrm{th}}+\varepsilon \ell_{\mathrm{p}} \frac{E_{\mathrm{th}}^{3}}{8 E_{\mathrm{IR}}} \simeq E_{\mathrm{th}-\mathrm{SR}}
$$

where $E_{\mathrm{IR}}$ is the background infrared energy (soft-photon energy) and $E_{\mathrm{th}}$ is the physical (amended) threshold energy. Nonetheless, we should also mention that there are some problems in the models of the DSR, such as the lack of a standard approach for achieving the DSR and not having a unique type of transformations of the spacetime, that have been considered in Ref. [174]. Moreover, it has

\footnotetext{
${ }^{24}$ For this subject; see, e.g., Ref. [185].
} 
been argued [187] that the DSR with an energy-dependent speed of light has some inconsistencies, and wherein, the present-day observations in particle physics rule out its first-order modification in the speed of light.

Now, let us consider our above expression about these two types of models. First, in the VSL models, those scalar (or vector) fields (that are introduced to describe the variation of the speed of light) essentially need to be assumed almost constants (or very slowly varying) over small cosmological intervals, for to comply with the recent cosmological considerations. Thus, these models are in no use for justification of the observed UHECR which are actually effective in those intervals. On the other hand, although the DSR has emerged as a VSL effective model, but it acts just for the observational implications of the UHECR [156, 162, 176, 188. Indeed, with the speed of light as a function of energy, it predicts the variation of the speed of light in the range of ultra high-energies. Thus, such a variation of the speed of light in the DSR is in no use for justification of the observed Webb redshift which indicates the variations of the speed of light in low-energies. Therefore, there is no single model for justification of these two observed phenomena via a single mechanism. In this regard, in another work [189, we have defined and introduced a new type of ether model, consistent with the Mach ideas, that can justify both of the observed phenomena.

\section{Acknowledgements}

We thank the Research Office of Shahid Beheshti University for the financial support.

\section{References}

[1] W. Rindler, "Relativity: Special, General and Cosmological", (Oxford University Press, Oxford, 2nd Ed. 2006).

[2] I. Newton, "Philosophice Naturalis Principia Mathematica", (Streater, London, 1st Ed. 1687), Final Ed. in English by: A. Motte, 1729, Revised by: A. Cajori, "Sir Isaac Newton's Mathematical Principles of Natural Philosophy and His System of the World', (University of California Press, Berkeley, 1962).

[3] F. Michael, "Leibniz's Metaphysics of Time and Space", (Springer, Heidelberg, 2008).

[4] R. Dean, "Symmetry, Structure and Spacetime", (Elsevier, Oxford, 2008).

[5] E. Erlichson, "The Leibniz-Clarke controversy: Absolute versus relative space and time", Am. J. Phys. 35 (1967), 89.

[6] M. Čapek [Editor], "The Concepts of Space and Time, Their Structure and Their Development", Boston Studies in The Philosophy of Science, Vol. 74, (Reidel Publishing Company, Boston, 1976).

[7] J. Earman and J. Norton, "What price spacetime substantivalism? The hole story", British J. Phil. Sci. 38 (1987), 515.

[8] G. Berkley, "De Motu or The principle and nature of motion and the cause of the communication of motions", (1721).

[9] J.C. Maxwell, "A dynamical theory of the electromagnetic field", Roy. Soc. 155 (1865), 459. This article accompanied a December 8, 1864 presentation by Maxwell to the Royal Society.

[10] I. Adler, "A New Look at Geometry", (John Day Company, NewYork, 1966).

[11] R.P. Hardie and R.K. Gaye [Translators], "The Works of Aristotle", Vol. 2 "Physica", (Clarendon Press, Oxford, 1930). 
[12] Aristotle, "Physics" (Oxford World's Classics), Edited by: D. Bostock, Translated by: R. Waterfield, (Oxford University Press, Oxford, 2008).

[13] B. Mashhoon, H. Liu and P.S. Wesson, "Space-time-matter", In: "Proceedings 7th Marcel Grossmann Meeting", Stanford (1994), pp. 333-335.

[14] R. d'Inverno, "Introducing Einstein's Relativity", (Clarendon Press, Oxford, 1992).

[15] M. Janssen, "Of pots and holes: Einstein's bumpy road to general relativity", Ann. Phys. (Berlin) 14 Supplement, (2005), 58.

[16] E. Mach, "La Meccanica nel suo Sviluppo Storico-Critico (Mechanics in Its Development Historical-Critical)", (Boringhieri, Torino, 1977), Italian translation from the original 9th German Ed. of 1933 (1st Ed. 1883). Also published as "The Science of Mechanics: A Critical and Historical Account of Its Development", (Open Court, Illinois, 1960).

[17] J. Barbour and H. Pfister [Editors], "Mach's Principle: From Newton's Bucket to Quantum Gravity", Einstein Studies, Vol. 6, (Birkhäuser, Boston, 1995).

[18] J. Lense and H. Thirring, "Über den Einfluss der Eigenrotation der Zentralkörper auf die Bewegung der Planeten und Monde nach der Einsteinschen Gravitationstheorie (About the influence of the self-rotation of cenral body to the movement of planets and moons according to Einstein's theory of gravitation)", Physik. Z. 19 (1918), 156.

[19] B. Mashhoon, F.W. Hehl and D.S. Theiss, "On the gravitational effects of rotating masses: The Thirring-Lense papers", Gen. Rel. Grav. 16 (1984), 711.

[20] I. Ciufolini, "The 1995-99 measurements of the Lense-Thirring effect using laser-ranged satellites", Class. Quant. Grav. 17 (2000), 2369.

[21] F. Everitt, et al., "Gravity Probe B: Final results of a space experiment to test general relativity", Phys. Rev. Lett. 106 (2011), 221101.

[22] L. Iorio, "Some considerations on the present-day results for the detection of frame-dragging after the final outcome of GP-B", Europhys. Lett. 96 (2011), 30001.

[23] A.P. French, "Special Relativity", (W.W. Norton, New York, 1966).

[24] R. Resnick, "Introduction to Special Relativity", (Wiley, New York, 1968).

[25] A.A. Michelson and E.W. Morley, "On the relative motion of the earth and the luminiferous ether", Am. J. Sci. 34 (1887), 333.

[26] R.S. Shankland, S.W. McCuskey, F.C. Leone and G. Kuerti, "New analysis of the interferometer observations of Dayton C. Miller", Rev. Mod. Phys. 27 (1955), 167.

[27] R.S. Shankland, "Michelson-Morley experiment", Am. J. Phys. 32 (1964), 16.

[28] F. Fitzgerald, "The ether and the earth's atmosphere", Sci. 13 (1889), 390.

[29] H.A. Lorentz, "La théorie électromagnétique de Maxwell et son application aux corps mouvants (The electromagnetic theory of Maxwell and its application to moving bodies)", Arch. Néerl. Sci. Ex. Nat. 25 (1892), 363.

[30] H.A. Lorentz, "The relative motion of the earth and the aether", Zitt. Akad. V. Wet. 1 (1892), 74. 
[31] H.A. Lorentz, "The Theory of Electrons and Its Applications to The Phenomena of Light and Radiatiant Heat", (Columbia University Press, New York, 1909; Dover Publications, New York, 1952).

[32] H.A. Lorentz, "Electromagnetic phenomena in a system moving with any velocity less than that of light", Proc. Acad. Sci. Amsterdam 6 (1904), 809. Reprinted in: "The Principle of Relativity: A Collection of Original Memoirs on The Special and General Theory of Relativity", by: H.A. Lorentz, A. Einstein, H. Minkowski and H. Weyl, Translated by: W. Perrett and G.B. Jeffery, (Dover Publications, New York, 1952), pp. 9-34.

[33] R.J. Kennedy and E.M. Thorndike, "Experimental establishment of the relativity of time", Phys. Rev. 42 (1932), 400.

[34] J. Bradley, "New discovered motion of the fixed stars", Phil. Trans. Roy. Soc. 35 (1727), 637.

[35] A.B. Stewart, "The discovery of stellar aberration", Sci. Am. 210 (March 1964), 100.

[36] A.A. Michelson and E.W. Morley, "Influence of motion of the medium on the velocity of light", Am. J. Sci. 31 (1886), 377.

[37] H.R. Bilger and W.K. Stowell, "Light drag in a ring laser: An improved determination of the drag coefficient", Phys. Rev. A 16 (1977), 313.

[38] G.A. Sanders and S. Ezekiel, "Measurement of Fresnel drag in moving media using a ring resonator technique", J. Opt. Soc. Am. B 5 (1988), 674.

[39] H.E. Ives, "Historical note on the rate of a moving atomic clock", J. Opt. Soc. Am. 37 (1947), 810.

[40] E.T. Whittaker, "A History of The Theories of Ether and Electricity: The Modern Theories 1900-1926", (Nelson, London, 1953; Harper, New York, 1960; Humanities Press, London, 1973).

[41] G. Holton, "On the origins of the special theory of relativity", Am. J. Phys. 28 (1960), 627.

[42] W. Rindler, "Einstein's priority in recognizing time dilation physically", Am. J. Phys. 38 (1970), 1111.

[43] H. Erlichson, "The rod contraction-clock retardation ether theory and the special theory of relativity", Am. J. Phys. 41 (1973), 1068.

[44] A.G. Riess, et al., "Observational evidence from supernovae for an accelerating universe and a cosmological constant", Astron. J. 116 (1998), 1009.

[45] S. Perlmutter, et al. [The Supernova Cosmology Project], "Measurements of Omega and Lambda from 42 high-redshift supernovae", Astrophys. J. 517 (1999), 565.

[46] A.G. Riess, et al., "BV RI light curves for 22 type Ia supernovae", Astron. J. 117 (1999), 707.

[47] A.G. Riess, et al., "Type Ia supernova discoveries at $z>1$ from the Hubble space telescope: Evidence for past deceleration and constraints on dark energy evolution", Astrophys. J. 607 (2004), 665 .

[48] A. Einstein, "Zur Elektrodynamik bewegter Körper", Ann. Phys. (Berlin) 322 (1905), 891. Its English version: "On the electrodynamics of moving bodies", In: "The Principle of Relativity: A Collection of Original Memoirs on The Special and General Theory of Relativity", by: H.A. Lorentz, A. Einstein, H. Minkowski and H. Weyl, Translated by: W. Perrett and G.B. Jeffery, (Dover Publications, New York, 1952), pp. 35-65. 
[49] H. Minkowski, "Raum und Zeit", Jber. Deutsch. Math.-Verein. 18 (1909), 75. Address delivered at the 80th Assembly of German Natural Scientists and Physicians, Cologne, Sept. 21, 1908. Its English version: "Space and time", In: "The Principle of Relativity: A Collection of Original Memoirs on The Special and General Theory of Relativity", by: H.A. Lorentz, A. Einstein, H. Minkowski and H. Weyl, Translated by: W. Perrett and G.B. Jeffery, (Dover Publications, New York, 1952), pp. 73-91.

[50] M. Born, "Die Theorie des starren Elektrons in der Kinematik des Relativitätsprinzips (The theory of rigid electron in the kinematics of principle of relativity)", Ann. Phys. (Berlin) 335 (1909), 1.

[51] P. Ehrenfest, "Gleichförmige Rotation starrer Körper und Relativitätstheorie (Uniform rotation of rigid bodies and theory of relativity.)", Physik. Z. 10 (1909), 918.

[52] V. Petkov, "Relativity and The Nature of Spacetime", (Springer, Berlin, 2005).

[53] A. Iorio, "Three questions on Lorentz violation", J. Phys. Conf. Ser. 67 (2007), 012008.

[54] D. Mattingly, "Modern tests of Lorentz invariance", Living Rev. Rel. 8 (2005), 5.

[55] K. Shinozaki, et al. [AGASA Collaboration], "AGASA results", Nucl. Phys. B 136 (2004), 18.

[56] F.W. Stecker, M.A. Malkan and S.T. Scully, "Intergalactic photon spectra from the far-IR to the UV Lyman limit for $0<z<6$ and the optical depth of the universe to high-energy gamma rays", Astrophys. J. 648 (2006), 774.

[57] R.U. Abbasi, et al., "First observation of the Greisen-Zatsepin-Kuzmin suppression", Phys. Rev. Lett. 100 (2008), 101101.

[58] J. Drożdżyński, "Evidence for an invalidity of the principle of relativity", J. Mod. Phys. 2 (2011), 1247.

[59] O. Sela, B. Tamir, S. Dolev and A.C. Elitzur, "Can special relativity be derived from Galilean mechanics alone?", Found. Phys. 39 (2009), 499.

[60] S. Coleman and S.L. Glashow, "High-energy tests of Lorentz invariance", Phys. Rev. D 59 (1999), 116008.

[61] A. Einstein, "Die Feldgleichungen der Gravitation (The field equations of gravitation)", Preuss. Akad. Wiss. Berlin Sitz. 17 (1915), 844.

[62] A. Einstein, "Die Grundlage der allgemeinen Relativitätstheorie", Ann. Phys. (Berlin) 354 (1916), 769. Its English version: "The foundation of the general theory of relativity", In: "The Principle of Relativity: A Collection of Original Memoirs on The Special and General Theory of Relativity", by: H.A. Lorentz, A. Einstein, H. Minkowski and H. Weyl, Translated by: W. Perrett and G.B. Jeffery, (Dover Publications, New York, 1952), pp. 109-164.

[63] H. Lichtenegger and B. Mashhoon, "Mach's principle", In: "The Measurment of Gravitomagnetism: A Challenging Enterprise", Edited by: L. Iorio, (NOVA Science, Hauppage, New York, 2005), pp. 13-27, arXiv: physics/0407078.

[64] I. Ciufolini and J.A. Wheeler, "Gravitation and Inertia", (Princeton University Press, Princeton, 1995).

[65] N. Straumann, "General Relativity With Applications to Astrophysics", (Springer, Berlin, 2004).

[66] J. Mehra, "Einstein, Hilbert, and The Theory of Gravitation", (Reidel Publishing Company, Holland, 1974). 
[67] A. Pais, "Subtle Is The Lord, The Science and The Life of Albert Einstein", (Oxford University Press, Oxford, 1982).

[68] J. Stachel, "Einstein's struggle with general covariance, 1912-1915", Presented at General Relativity and Gravitation 9th, 1980 at Jena, Germany; Reprinted as "Einstein's search for general covariance, 1912-1915", In: "Einstein and The History of General Relativity", based on the Proceedings of May 1986, Osgood Hill Conference, Massachusetts, Edited by: D. Howard and J. Stachel, (The Center for Einstein Studies, Boston University, 1989), pp. 63-100.

[69] J. Stachel, "What a physicist can learn from the discovery of general relativity", In: "Proceedings of The Fourth Marcel Grossmann Meeting on General Relativity", Edited by: R. Ruffini, (NorthHolland, Amsterdam, 1986), pp. 1857-1862.

[70] J. Norton, "How Einstein found his field equations, 1912-1915", In: "Einstein and The History of General Relativity", based on the Proceedings of May 1986, Osgood Hill Conference, Massachusetts, Edited by: D. Howard and J. Stachel, (The Center for Einstein Studies, Boston University, 1989), pp. 101-159. It is reprinted from "Historical Studies in The Physical Sciences", Vol. 14, Part 2, Edited by: J.L. Heilbron, (The Regents of The University of California, Berkeley, 1984), pp. 253-316.

[71] J.L. Synge, "Relativity: The General Theory", (North-Holland, Amsterdam, 1960).

[72] N.D. Birrell and P.C.W. Davies, "Quantum Fields in Curved Space", (Cambridge University Press, Cambridge, 1982).

[73] I.L. Buchbinder, S.D. Odintsov and I.L. Shapiro, "Effective Action in Quantum Gravity", (Institute of Physics Publishing, Bristol, 1992).

[74] A. Einstein and M. Grossmann, "Entwurf einer verallgemeinerten Relativitätstheorie und einer Theorie der Gravitation (Draft of a generalized relativity theory and a theory of gravitation)", $Z$. Math. Phys. 62 (1913), 225.

[75] A. Einstein and M. Grossmann, "Kovarianzeigenschaften der Feldgleichungen der auf die verallgemeinerte Relativitätstheorie gegründeten Gravitationstheorie (Covariance properties of the field equations of the gravitational theory based on generalized relativity)", Z. Math. Phys. 63 (1914), 215.

[76] A. Einstein wrote to: P. Ehrenfest, on 26th December, 1915, EA 9-363.

[77] A. Einstein wrote to: M. Besso, on 3rd January, 1916, In: "Albert Einstein, Michele Besso Correspondence 1903-1955", Edited by: P. Speziali, (Hermann, Paris, 1972), pp. 63-64.

[78] A. Einstein, "Relativity and The Problem of Space (1952)", Appendix 5, In: "Relativity, The Special and The General Theory: A Popular Exposition", Translated by: R.W. Lawson, (Methuen, London, 15th Ed. 1954), pp. 135-157.

[79] A.S. Eddington, “'Space' or 'Æther'?”, Nature 107 (1921), 201.

[80] A. Einstein, "Äther und Relativitätstheorie (Ether and Relativity Theory)", (Springer, Berlin, 1920), reprinted as "Sidelights on Relativity", (Dover Publications, New York, 1983).

[81] A. Trautman, "Comparison of Newtonian and relativistic theories of space-time", In: "Perspectives in Geometry and Relativity", Edited by: B. Hoffmann, (Indiana University Press, Bloomington, 1966), pp. 413-425.

[82] E.T. Whittaker, "A History of The Theories of Ether and Electricity: The Classical Theories", (Nelson, London, 2nd Ed. 1951; Tomash Publishers, New York, 1987). 
[83] K.F. Schaffner, "Nineteenth-Century Ether Theories", (Pergamon Press, New York, 1972).

[84] M.J. Dupré and F.J. Tipler, "General relativity as an æther theory", Int. J. Mod. Phys. D 21 (2012), 1250011.

[85] R. Gautreau, "Newton's absolute time and space in general relativity", Am. J. Phys. 68 (2000), 350.

[86] D. Savickas, "General relativity exactly described in terms of Newton's laws within curved geometries", Int. J. Mod. Phys. D 23 (2014), 1430018.

[87] A. Salam, "Gauge unification of fundamental forces", Rev. Mod. Phys. 52 (1980), 525.

[88] P.S. Wesson and J. Ponce de Leon, "Kaluza-Klein equations, Einstein's equations, and an effective energy-momentum tensor", J. Math. Phys. 33 (1992), 3883.

[89] C. Romero, R. Tavakol and R. Zalaletdinov, "The embedding of general relativity in five dimensions", Gen. Rel. Grav. 28 (1996), 365.

[90] J.M. Overduin and P.S. Wesson, "Kaluza-Klein gravity", Phys. Rep. 283 (1997), 303.

[91] P.S. Wesson, "Space-Time-Matter: Modern Kaluza-Klein Theory", (World Scientific, Singapore, 1999).

[92] P.S. Wesson, "Five-Dimensional Physics: Classical and Quantum Consequences of Kaluza-Klein Cosmology", (World Scientific, Singapore, 2006).

[93] A.F. Bahrehbakhsh, M. Farhoudi and H. Shojaie, "FRW cosmology from five dimensional vacuum Brans-Dicke theory", Gen. Rel. Grav. 43 (2010), 847.

[94] S.M.M. Rasouli, M. Farhoudi and H.R. Sepangi "Anisotropic cosmological model in modified Brans-Dicke theory", Class. Quant. Grav. 28 (2011), 155004.

[95] A.F. Bahrehbakhsh, M. Farhoudi and H. Vakili, "Dark energy from fifth dimensional BransDicke theory", Int. J. Mod. Phys. D 22 (2013), 1350070.

[96] S.M.M. Rasouli, M. Farhoudi and P.V. Moniz, "Modified Brans-Dicke theory in arbitrary dimensions", Class. Quant. Grav. 31 (2014), 115002.

[97] T. Harko, "Modified gravity with arbitrary coupling between matter and geometry", Phys. Lett. B 669 (2008), 376.

[98] T. Harko, F.S.N. Lobo, S. Nojiri and S.D. Odintsov, " $f(R, T)$ gravity", Phys. Rev. D 84 (2011), 024020 .

[99] Y. Bisabr, "Modified gravity with a nonminimal gravitational coupling to matter", Phys. Rev. D 86 (2012), 044025.

[100] M. Jamil, D. Momeni, R. Muhammad and M. Ratbay, "Reconstruction of some cosmological models in $f(R, T)$ gravity", Eur. Phys. J. C $\mathbf{7 2}$ (2012), 1999.

[101] F.G. Alvarenga, A. de la Cruz-Dombriz, M.J.S. Houndjo, M.E. Rodrigues and D. Saez-Gomez, "Dynamics of scalar perturbations in $f(R, T)$ gravity", Phys. Rev. D 87 (2013), 103526.

[102] Z. Haghani, T. Harko, F.S.N. Lobo, H.R. Sepangi and S. Shahidi, "Further matters in spacetime geometry: $f\left(R, T, R_{\mu \nu} T^{\mu \nu}\right)$ gravity", Phys. Rev. D 88 (2013), 044023.

[103] H. Shabani and M. Farhoudi, " $f(R, T)$ cosmological models in phase-space", Phys. Rev. D 88 (2013), 044048. 
[104] H. Shabani and M. Farhoudi, "Cosmological and solar system consequences of $f(R, T)$ gravity models", Phys. Rev. D 90 (2014), 044031.

[105] R. Zaregonbadi and M. Farhoudi, "Late time acceleration from matter-curvature coupling", submitted to journal.

[106] A. Einstein, "Kosmologische Betrachtungen zur allgemeinen Relativitätstheorie", Preuss. Akad. Wiss. Berlin, Sitz. (1917), 142. Its English version: "Cosmological considerations on the general theory of relativity", In: "The Principle of Relativity: A Collection of Original Memoirs on The Special and General Theory of Relativity", by: H.A. Lorentz, A. Einstein, H. Minkowski and H. Weyl, Translated by: W. Perrett and G.B. Jeffery, (Dover Publications, New York, 1952), pp. $175-188$.

[107] W. de Sitter, "On the curvature of space", Proc. Kon. Ned. Acad. Wet. 20 (1918), 229.

[108] E.P. Hubble, "A relation between distance and radial velocity among extragalactic nebulae", Proc. Nat. Acad. Sci. USA 15 (1929), 169.

[109] G. Gamow, "My World Line, An Informal Autobiography", (Viking, New York, 1970).

[110] A. Einstein wrote to: F. Pirani, 1954, EA 17-448.

[111] N. Namavarian and M. Farhoudi, "Cosmological constant implementing Mach principle in general relativity", submitted to journal.

[112] S. Weinberg, "The cosmological constant problem", Rev. Mod. Phys. 61 (1989), 1.

[113] S.M. Carroll, "The cosmological constant", Living. Rev. Rel. 4 (2001), 1.

[114] V. Sahni, "The cosmological constant problem and quintessence", Class. Quant. Grav. 19 (2002), 3435.

[115] S. Nobbenhuis, "Categorizing different approaches to the cosmological constant problem", Found. Phys. 36 (2006), 613.

[116] H. Padmanabhan and T. Padmanabhan, "CosMIn: The solution to the cosmological constant problem", Int. J. Mod. Phys. D 22 (2013), 1342001.

[117] D. Bernard and A. LeClair, "Scrutinizing the cosmological constant problem and a possible resolution", Phys. Rev. D 87 (2013), 063010.

[118] S. Weinberg, "Cosmology", (Oxford University Press, Oxford, 2008).

[119] J.D. Barrow and D.J. Shaw, "The value of the cosmological constant", Gen. Rel. Grav. 43 (2011), 2555.

[120] P.J.E. Peebles and B. Ratra, "The cosmological constant and dark energy", Rev. Mod. Phys. 75 (2003), 559.

[121] T. Padmanabhan, Cosmological constant-the weight of the vacuum, Phys. Rep. 380 (2003), 235.

[122] D. Polarski, "Dark energy: Current issues", Ann. Phys. (Berlin) 15 (2006), 342.

[123] E.J. Copeland, M. Sami and S. Tsujikawa, "Dynamics of dark energy", Int. J. Mod. Phys. D 15 (2006), 1753.

[124] R. Durrer and R. Maartens, "Dark energy and dark gravity: Theory overview", Gen. Rel. Grav. 40 (2008), 301. 
[125] K. Bamba, S. Capozziello, S. Nojiri and S.D. Odintsov, "Dark energy cosmology: The equivalent description via different theoretical models and cosmography tests", Astrophys. Space Sci. 342 (2012), 155 .

[126] P.A.R. Ade, et al. [Planck Collaboration], "Planck 2013 results. XVI. Cosmological parameters", Astron. Astrophys. 571 (2014), A16.

[127] P.A.R. Ade, et al. [Planck Collaboration], "Planck 2015 results. XIII. Cosmological parameters", arXiv: 1502.01589.

[128] M. Farhoudi, "On higher order gravities, their analogy to GR, and dimensional dependent version of Duff's trace anomaly relation", Gen. Rel. Grav. 38 (2006), 1261.

[129] G. Bertonea, D. Hooperb and J. Silk, "Particle dark matter: Evidence, candidates and constraints", Phys. Rep. 405 (2005), 279.

[130] J. Silk, "Dark matter and galaxy formation", Ann. Phys. (Berlin) 15 (2006), 75.

[131] J.L. Feng, "Dark matter candidates from particle physics and methods of detection", Annu. Rev. Astron. Astrophys. 48 (2010), 495.

[132] L. Bergström, "Dark matter evidence, particle physics candidates and detection methods", Ann. Phys. (Berlin) $\mathbf{5 2 4}$ (2012), 479.

[133] C.S. Frenk and S.D.M. White, "Dark matter and cosmic structure", Ann. Phys. (Berlin) 524 (2012), 507.

[134] M. Farhoudi, "Classical trace anomaly", Int. J. Mod. Phys. D 14 (2005), 1233.

[135] C. Brans and R.H. Dicke, "Mach's principle and a relativistic theory of gravitation", Phys. Rev. 124 (1961), 925.

[136] R.H. Dicke, "Mach's principle and invariance under transformation of units", Phys. Rev. 125 (1962), 2163.

[137] Y. Fujii and K. Maeda, "The Scalar-Tensor Theory of Gravitation", (Cambridge University Press, Cambridge 2004).

[138] H. Farajollahi, M. Farhoudi and H. Shojaie, "On dynamics of Brans-Dicke theory of gravitation", Int. J. Theor. Phys. 49 (2010), 2558.

[139] V. Faraoni, "Cosmology in Scalar Tensor Gravity", (Kluiwer Academic Publishers, Netherlands, 2004).

[140] S. Capozziello and V. Faraoni, "Beyond Einstein Gravity: A Survey of Gravitational Theories for Cosmology and Astrophysics", (Springer, Heidelberg, 2011).

[141] J. Khoury and A. Weltman, "Chameleon cosmology", Phys. Rev. D 69 (2004), 044026.

[142] P. Brax, C. Burrage, A.-C. Davis, D. Seery and A. Weltman, "Higgs production as a probe of chameleon dark energy", Phys. Rev. D 81 (2010), 103524.

[143] H. Farajollahi, M. Farhoudi, A. Salehi and H. Shojaie, "Chameleonic generalized Brans-Dicke model and late-time acceleration", Astrophys. Space Sci. 337 (2012), 415.

[144] N. Saba and M. Farhoudi, "Chameleonic inflation in the light of Planck 2015", work in progress.

[145] T. Jacobson and D. Mattingly, "Gravity with a dynamical preferred frame", Phys. Rev. D 64 (2001), 024028. 
[146] C. Eling, T. Jacobson and D. Mattingly, "Einstein-æther theory", arXiv: $g r-q c / 0410001$.

[147] T. Jacobson, "Einstein-æther gravity: A status report", PoS QG-Ph (2007), 020, arXiv: $0801.154 \%$

[148] J.D. Barrow, "Some inflationary Einstein-aether cosmologies", Phys. Rev. D 85 (2012), 047503.

[149] H. Wei, X.-P. Yan and Y.-N. Zhou, "Cosmological evolution of Einstein-aether models with power-law-like potential", Gen. Rel. Grav. 46 (2014), 1719.

[150] Z. Haghani, T. Harko, H.R. Sepangi and S. Shahidi, "Scalar Einstein-aether theory", arXiv: 1404.7689 .

[151] C. Furtado, J.R. Nascimento, A.Y. Petrov and A.F. Santos, "The æther-modified gravity and the Gödel metric", arXiv: 1109.5654.

[152] "The Timaeus of Plato", Edited with Introduction and Notes by: R.D. Archer-Hind, (Macmillan, London, 1888).

[153] Plato, "Timaeus", Translated by: B. Jowett, (Echo Library, United Kingdom, 2006).

[154] J.K. Webb, et al., "A search for time variation of the fine structure constant", Phys. Rev. Lett. 82 (1999), 884.

[155] M.T. Murphy, et al., "Possible evidence for a variable fine structure constant from QSO absorption lines: Motivations, analysis and results", Mon. Not. Roy. Astron. Soc. 327 (2001), 1208.

[156] J. Albert, et al. [MAGIC Collaboration], "Probing quantum gravity using photons from a flare of the active galactic nucleus Markarian 501 observed by the MAGIC telescope", Phys. Lett. B 668 (2008), 253.

[157] J.D. Barrow and J. Magueijo, "Varying $-\alpha$ theories and solutions to the cosmological problems", Phys. Lett. B 443 (1998), 104.

[158] H.B. Sandvik, J.D. Barrow and J. Magueijo, "A simple varying-alpha cosmology", Phys. Rev. Lett. 88 (2002), 031302.

[159] J.D. Barrow and J. Magueijo, "Solutions to the quasi-flatness and quasi-lambda problems", Phys. Lett. B 447 (1998), 246.

[160] M.A. Clayton and J.W. Moffat, "Dynamical mechanism for varying light velocity as a solution to cosmological problem", Phys. Lett. B 480 (1998), 263.

[161] A. Albrecht and J. Magueijo, "A time varying speed of light as a solution to cosmological puzzles", Phys. Rev. D 59 (1999), 043516.

[162] J. Magueijo, "New varying speed of light theories", Rep. Prog. Phys. 66 (2003), 2025.

[163] H. Shojaie and M. Farhoudi, "A cosmology with variable c", Can. J. Phys. 84 (2006), 933.

[164] H. Shojaie and M. Farhoudi, "A varying-c cosmology", Can. J. Phys. 85 (2007), 1395.

[165] J. Magueijo, J.D. Barrow and H.B. Sandvik, "Is it e or is it c? Experimental tests of varying alpha", Phys. Lett. B 549 (2002), 284.

[166] P. Castorina and D. Zappala, "Noncommutative electrodynamics and ultra high energy gamma rays", Europhys. Lett. 64 (2003), 641. 
[167] R. Horvat, D. Kekez, P. Schupp, J. Trampeti and J. You, "Photon-neutrino interaction in $\theta$-exact covariant noncommutative field theory", Phys. Rev. D 84 (2011), 045004.

[168] G. Amelino-Camelia, "Relativity in space-times with short-distance structure governed by an observer-independent (Planckian) length scale", Int. J. Mod. Phys. D 11 (2002), 35.

[169] J. Magueijo and L. Smolin, "Lorentz invariance with an invariant energy scale", Phys. Rev. Lett. 88 (2002), 190403.

[170] J. Kowalski-Glikman and S. Nowak, "Non-commutative space-time of doubly special relativity theories", Int. J. Mod. Phys. D 12 (2003), 299.

[171] H.-Y. Guo, C.-G. Huang, Z. Xu and B. Zhou, "On de Sitter invariant special relativity and cosmological constant as origin of inertia", Mod. Phys. Lett. A 19 (2004), 1701.

[172] A. Agostini, G. Amelino-Camelia and F. D'Andrea, "Hopf-algebra description of noncommutative-spacetime symmetries", Int. J. Mod. Phys. A 19 (2004), 5187.

[173] H.-Y. Guo, H.-T. Wu and B. Zhou, "The principle of relativity and the special relativity triple", Phys. Lett. B 670 (2009), 437.

[174] G. Amelino-Camelia, "Doubly-special relativity: Facts, myths and some key open issues", Symmetry 2 (2010), 230.

[175] S. Alexander, "On the varying speed of light in a brane-induced FRW universe", J. High Energy Phys. 0011 (2000), 017.

[176] I.T. Drummond and S.J. Hathrell, "QED vacuum polarization in a background gravitational field and its effect on the velocity of photons", Phys. Rev. D 22 (1980), 343.

[177] M.A. Clayton and J.W. Moffat, "Dynamical mechanism for varying light velocity as a solution to cosmological problems", Phys. Lett. B 460 (1999), 263.

[178] M.A. Clayton and J.W. Moffat, "Scalar-tensor gravity theory for dynamical light velocity", Phys. Lett. B 477 (2000), 269.

[179] J. Magueijo, "Bimetric varying speed of light theories and primordial fluctuations", Phys. Rev. D 79 (2009), 043525.

[180] D. Finkbeiner, M. Davis and D. Schlegel, "Detection of a far IR excess with DIRBE at 60 and 100 microns", Astrophys. J. 544 (2000), 81.

[181] D. Mazin and M. Raue, "New limits on the density of the extragalactic background light in the optical to the far infrared from the spectra of all known TeV blazars", Astron. Astrophys. 471 (2007), 439 .

[182] A.A. Penzias and R.H. Wilson, "A measurement of excess antenna temperature at $4080 \mathrm{Mc} / \mathrm{s}$ ", Astrophys. J. 142 (1965), 419.

[183] K. Greisen, "End to the cosmic-ray spectrum", Phys. Rev. Lett. 16 (1966), 748.

[184] G.T. Zatsepin and V.A. Kuzmin, "Upper limit of the spectrum of cosmic rays", J. Exp. Theor. Phys. Lett. 4 (1966), 78.

[185] S. Majid, "Foundations of Quantum Group Theory", (Cambridge University Press, Cambridge, 2000).

[186] V.A. Fock, "The Theory of Space-Time and Gravitation", (Pergamon Press, New York, 1964). 
[187] S. Hossenfelder, "The box-problem in deformed special relativity", arXiv: 0912.0090.

[188] S.T. Scully and F.W. Stecker, "Lorentz invariance violation and the observed spectrum of ultrahigh energy cosmic rays", Astropart. Phys. 31 (2009), 220.

[189] M. Yousefian and M. Farhoudi, "Justification of Webb's redshift and ultra high energy cosmic rays via an ether model", work in progress. 